\title{
Pleomorphic adenoma of the breast
}

\author{
Iulian Radu ${ }^{1,2}$, Ioana Petcu ${ }^{1, *}$, Andrian Pănuță ${ }^{1}$, Dragoș Scripcariu ${ }^{1,2}$, Mihaela Buna- \\ Arvinte $^{1}$, Karina Bilavschi ${ }^{3}$, Viorel Scripcariu ${ }^{1,2}$ \\ ${ }^{1}$ Department of General Surgery Regional Oncology Institute, lasi, Romania, ${ }^{2}$ "Grigore T. Popa” \\ University of Medicine and Pharmacy lasi, ${ }^{3}$ Department of Pathology Regional Oncology Institute, lasi, \\ Romania
}

\begin{abstract}
Pleomorphic adenoma, a common salivary gland tumor, is a rare benign breast tumor. It doesn't have specific features and its diagnosis can be made on the final histopathological examination. There have been reported few cases of malignant transformation and many recurrences, therefore it is required an adequate excision of this tumor with clear margins. We present the case of a woman of 47 years old, who was admitted in our Department for a right perimamelonar lump, with uncertain imaging features. A right mammary segmentectomy was performed and the routine histopathological and immunohistochemical examination led to the diagnosis of pleomorphic adenoma of the breast.
\end{abstract}

Keywords: pleomorphic adenoma of the breast, rare benign tumor

\section{Introduction}

Pleomorphic adenoma (PA) or benign mixed tumor (myoepithelial and epithelial cells embedded in chondroid stroma) [1] is a common salivary gland tumor, but a rare tumor type of the breast. Other uncommon sites of this kind of tumor are in the skin (chondroid syringoma), nasal septum, palate, larynx, paranasal sinuses and vulva $[2,3]$.

Clinically, PA presents as a palpable juxtaareolar breast lump which is difficult to differentiate from cancer [4]. The diagnosis of this type of tumor is made post operatively, on the final histopathological examination, because it has nonspecific features on ultrasound breast imaging and mammography.

PA has a pseudopod like extension into

Received: August 2016; Accepted after review: December 2016; Published: December 2016.

*Corresponding author: loana Petcu MD, Ist Surgical Unit, Regional Institute of Oncology lasi, 2-4, Gen. Henri Mathias Berthelot Street, 700483, Iasi, Romania

Email: iopet3@yahoo.com adjacent tissue and this is why it should be performed an adequate excision of the tumor with clear margins, so there would be no recurrence [4].

Three cases of carcinoma ex-pleomorphic adenoma of the breast associated with adjacent areas of pleomorphic adenoma, were reported by Hayes et al. [5], suggesting the potential of malignant transformation, but metastasis associated with the PA of the breast has not been reported, so far $[4,5]$.

\section{Case report}

We report the case of a woman of 47 years old, known with paroxysmal supraventricular tachycardia, atrial and ventricular extrasystoles, that presented to 1st Department of Surgery of the Regional Institute of Oncology lasi, for a right breast tumor with an uncertain diagnosis on the ultrasound breast imaging and mammography.

By admission in our clinic, the patient had no significant biological anomalies. The measured tumor marker of the breast, CA15.3 
was 28.2 U/ML and a chest X-ray showed no progressive lesions at the pulmonary and pleural level.

The ultrasound examination (Figure 1) reveals in the right breast, at the union of both exterior quadrants and at $3-4 \mathrm{~cm}$ away from the nipple, a hypoechoic and heterogeneous mass (with hyper and anechoic areas), oval shaped and measuring $16 \times 9 \times 10 \mathrm{~mm}$, lobulated, without Doppler Signal and with no visible right axillary adenopathy.
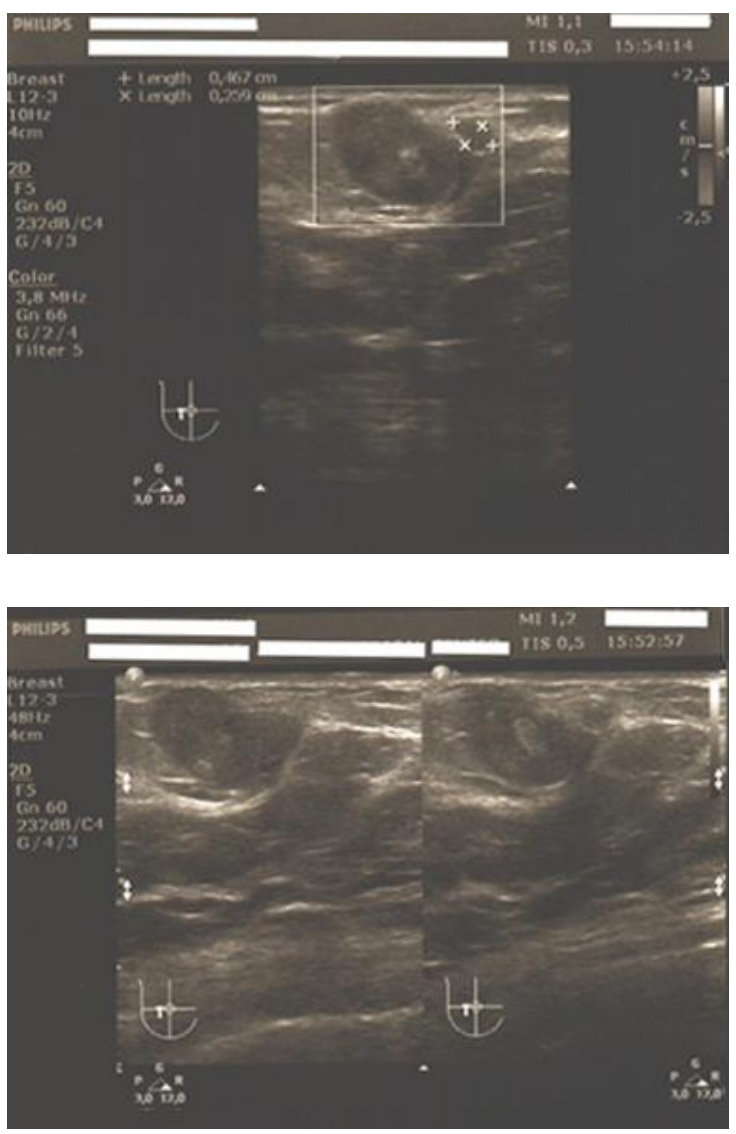

Fig. 1. Breast ultrasound: extensive pneumatosis, railroad tracks-like (arrows) and dilated bowel loops. No signs of pneumoperitoneum.

The mammography of the right breast showed an external perinipple nodular opacity of $15 / 10 \mathrm{~mm}$, with partially net outline, with several amorphous calcifications (Figure 2).
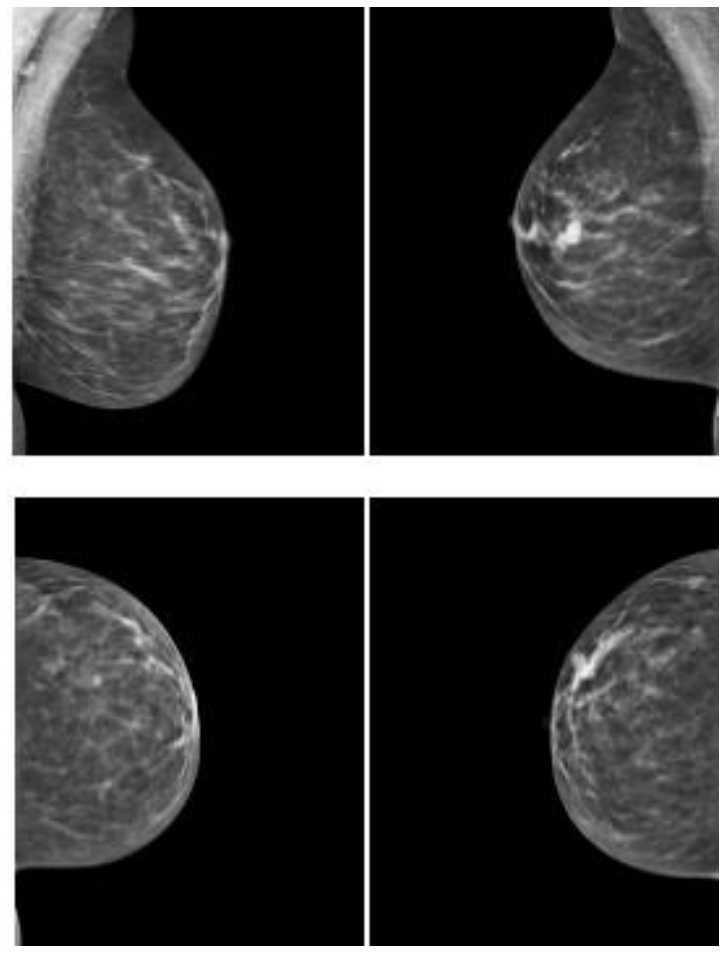

Fig. 2. The right breast mammography revealed a perinipple nodular opacity of $15 \times 10 \mathrm{~mm}$ with partially net outline and several amorphous calcifications.

We performed a right mammary segmentectomy (at patient's request) and sent the specimen for intraoperative frozen section diagnosis, which revealed an intracystic papillary carcinoma. The surgical specimen was subsequently submitted for paraffin embedding protocol.

The postoperative course of the patient was uneventful.

The histopathological examination (Figures $3 a$ and $3 b$ ) revealed a well differentiated tumor proliferation harboring a reduced cytonuclear atypia, with glandular, cribriform and trabecular architecture, and no obvious tumor necrosis. The immunohistochemical tests (Figures 4a-4e) confirmed both ductal and myoepithelial cells, hence the final histopathological diagnosis was pleomorphic adenoma. There were no extensions in the surrounding tissue and also no necrosis or lympho-vascular invasion were identified. 

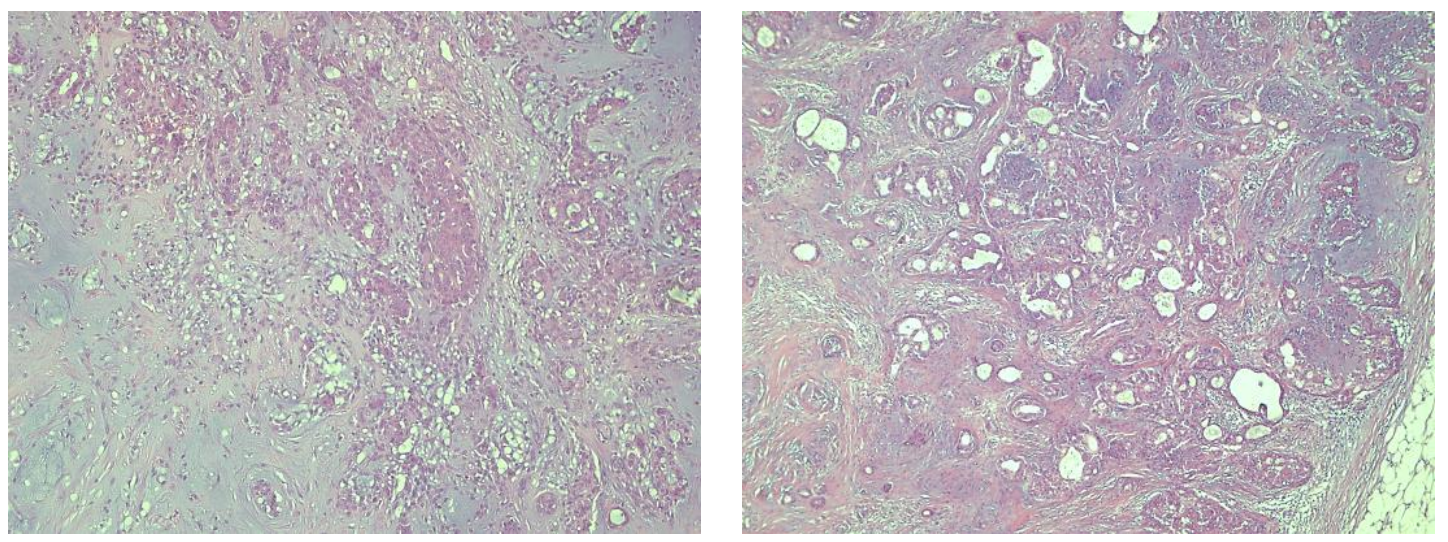

Fig. 3. Glandular, cribriform, and trabecular pattern of the tumor a) HE, $x 50$ and b) HE, $x 100$.

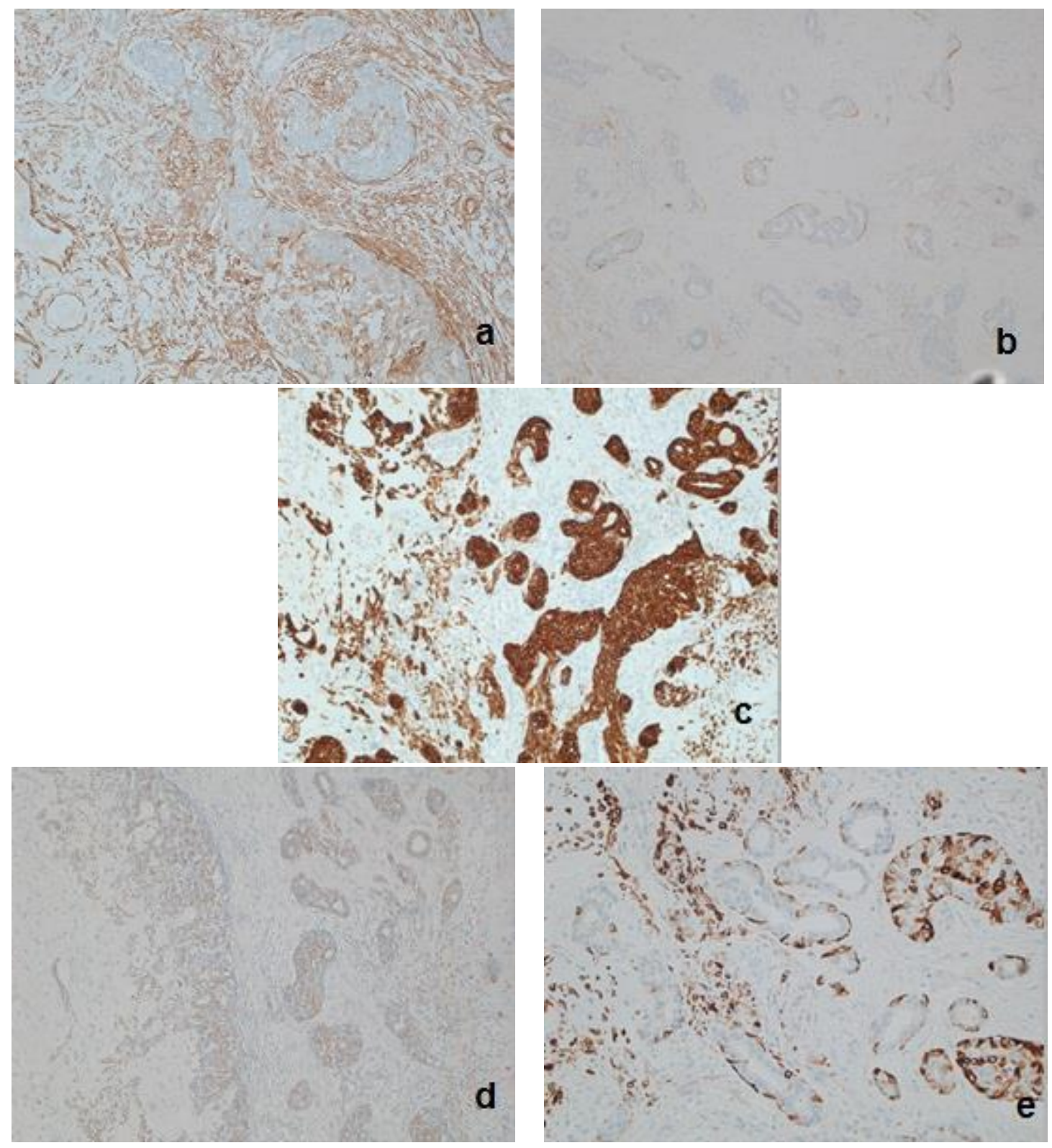

Fig. 4. Immunohistochemical expressions of the tumor cells for: a) Ab anti-H-caldesmon, $\mathrm{x} 100$; $b$ ) Ab antiSmooth Muscle Actin, x100; c) Ab anti-CK7, x100; d) Ab anti-S100, x100; e) Ab anti-GFAP, x200. 


\section{Discussions}

Pleomorphic adenoma is a rare tumor of the breast. It presents as a palpable juxtaareolar breast mass and it has nonspecific features on imaging examination. The final diagnosis is made on the histopathological findings.

There are many recurrences reported and few malignancies, based on the behavior of this kind of tumor and on its pseudopod like extension in the adjacent tissue. It is necessary to take into account the excision of the tumor with adequate margins, avoiding the radical mastectomy, because pleomorphic adenoma is not a malignant tumor. However, it is quite difficult to differentiate a pleomorphic adenoma from breast carcinoma using only the imaging findings that is why the final diagnosis is given by the histopathological examination.

Some papers suggest that the juxtaareolar location of the tumor and the circumscription could be the result of a large breast duct origin of the tumor [6].

So far, we could not find any reported metastasis of pleomorphic adenoma of the breast [2].

The main recommended treatment in the literature for PA is the excision of the tumor with clear margins, but there are also 3 cases of inevitable mastectomy reported, as far as we know [7].

Based on the histopathological findings (small tumor size, well defined, with reduced nuclear atypia, without obvious tumor necrosis), there are few differential diagnosis of pleomorphic adenoma of the breast, such as: adenocarcinoma with cartilage/osseous

\section{References}

1. Ginter PS, Scognamiglio $T$, Tauchi-Nishi $P$, et al. Pleomorphic adenoma of breast: a radiological and pathological study of a common tumor in an uncommon location. Case Rep Med 2015; Article ID 172750.

2. Khamechian $\mathrm{T}$, Alizargar J, Mazoochi $\mathrm{T}$. Reporting a rare case of pleomorphic adenoma of the breast. Case Rep Med 2014; Article ID 387183. metaplasia, stromal sarcoma, phyllodes tumor and fibroadenoma [2, 8].

There are also some diagnostic difficulties on frozen section because these tumors differentiate along epithelial and myoepithelial lines in different amounts, from case to case [9].

Even though there are less than 100 cases of pleomorphic adenoma of the breast declared, we should take into account this kind of tumor when we have a palpable juxtaareolar breast mass with uncertain imaging features. The best surgical treatment is to avoid radical mastectomy and to excise enough so there would be no recurrences or malignant transformation [10].

\section{Conclusion}

The particularity of our case consists in the differences between the imaging diagnosis that could not rule out a malignant tumor, the intraoperative frozen section diagnosis of a intracystic papillary carcinoma and the final histopathological diagnosis that revealed a pleomorphic adenoma.

\section{Consent}

Written informed consent was obtained from the patient for publication of this case report and accompanying images.

\section{Conflict of interest}

The author(s) declare that they have no competing interests.

3. Su A, Apple SK, Moatamed NA. Pleomorphic adenoma of the vulva, clinical reminder of a rare occurrence. Rare Tumors 2012; 4(1):e16.

4. Leekha $N$, Muralee $M$, Mathews $A$, et al. Pleomorphic adenoma of breast- a case report and review of literature. Indian J Surg Oncol 2014; 5(2):152-154.

5. Hayes MM, Lesack D, Girardet C, et al. Carcinoma ex-pleomorphic adenoma of the breast. Report of three cases suggesting a 
relationship to metaplastic carcinoma of matrixproducing type. Virchows Arch 2005; 446(2):142-149.

6. Chen KTK. Pleomorphic adenoma of the breast. Am J Clin Pathol 1990; 93(6):792-794.

7. John BJ, Griffiths C, Ebbs SR. Pleomorphic adenoma of the breast should be excised with a cuff of normal tissue. Breast $J$ 2007; 13(4):418-420.

8. Djakovic A, Engel JB, Geisinger E, Honig A, et al. Pleomorphic adenoma of the breast initially misdiagnosed as metaplastic carcinoma in preoperative stereotactic biopsy: a case report and review of the literature. Eur J Gynecol Oncol 2011; 32(4):427-430.

9. Foschini MP, Krausz T. Salivary gland-type tumors of the breast: a spectrum of benign and malignant tumors including 'triple negative carcinomas' of low malignant potential. Semin Diagn Pathol 2010; 27(1):77-90.

10. Sato K, Ueda $Y$, Shimasaki $M$, et al. Pleomorphic adenoma (benign mixed tumor) of the breast: a case report and review of the literature. Pathol Res Pract 2005; 201(4):333339. 